\title{
THE DYNAMIC BEHAVIOR OF WATER TANKS
}

\author{
By George W. Housner
}

\begin{abstract}
During the Chilean earthquakes of May, 1960, a number of large elevated water tanks were severely damaged whereas others survived without damage. An analysis of the dynamic behavior of such tanks must take into account the motion of the water relative to the tank as well as the motion of the tank relative to the ground. Some simple expressions are given for the pertinent dynamic properties of tanks with free water surface. A simplified dynamic analysis is indicated for the response of elevated water tanks to earthquake ground motion.
\end{abstract}

\section{INTRODUCTION}

The performance of elevated water tanks during earthquakes is of much interest to engineers, not only because of the importance of these tanks in controlling fires, but also because the simple structure of an elevated tank is relatively easy to analyze and, hence, the study of tanks can be informative as to the behavior of structures during earthquakes. During the Chilean earthquakes of May, 1960 a number of elevated water tanks were badly damaged as described in a companion paper by K. V. Steinbrugge and R. Flores. Other elevated water tanks survived without damage, as described in a companion paper by W. K. Cloud. A dynamic analysis of such tanks must take into account the motion of the water relative to the tank as well as the motion of the tank relative to the ground. If a closed tank is completely full of water or completely empty, it is essentially a one-mass structure. If, as is usual, the tank has a free water surface there will be sloshing of the water during an earthquake and this makes the tank essentially a two-mass structure. In this case, the dynamic behavior of an elevated tank may be quite different. For certain proportions of the tank and the structure the sloshing of the water may be the dominant factor, whereas for other proportions the sloshing may have small effect. Therefore, an understanding of the earthquake damage, or survival, of elevated water tanks requires an understanding of the dynamic forces associated with the sloshing water.

\section{Tank on the Ground}

A water tank or reservoir on the ground will have its contents excited into sloshing by an earthquake and the amplitude of the sloshing is indicative of the intensity of the ground motion. If a tank with a free water surface (fig. 1a) is subjected to horizontal ground acceleration $a$ the forces exerted on the tank by the water are of two kinds. First, when the walls of the tank accelerate back and forth a certain fraction of the water is forced to participate in this motion, which exerts a reactive force on the tank the same as would be exerted by a mass $M_{0}$ that is attached rigidly to the tank at the proper height as shown in figure $1 \mathrm{~b}$. The mass $M_{0}$ is attached at a height $h_{0}$ so that the horizontal force exerted by it is collinear with the resultant force exerted by the equivalent water. Second, the motion of the tank walls excites the water into oscillations which in turn exert an oscillating force on the tank. This oscillating force is the same as would be exerted by a mass $M_{1}$ that 
can oscillate horizontally against a restraining spring as shown in figure 1b. The mass $M_{1}$ corresponds to the fundamental mode of oscillation of the water which is the mode of importance for most earthquake problems. If the equivalent system shown in fig. $1 b$ is subjected to earthquake ground accelerations $a$, the forces exerted

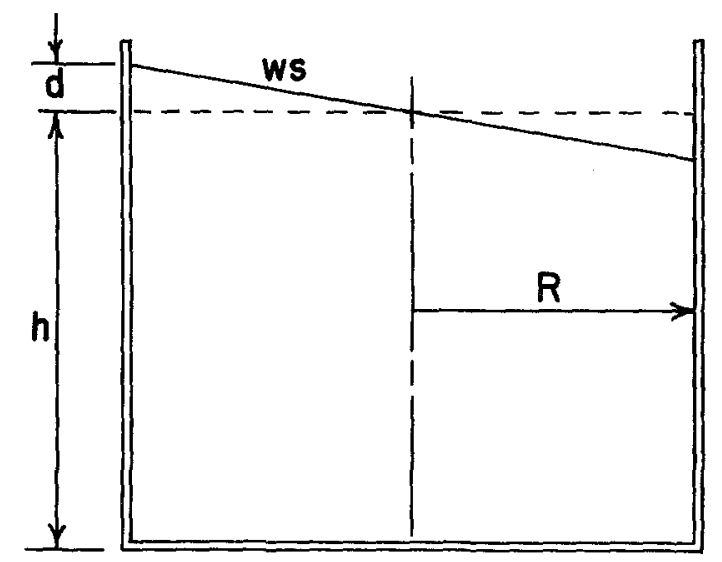

Fia, 1a

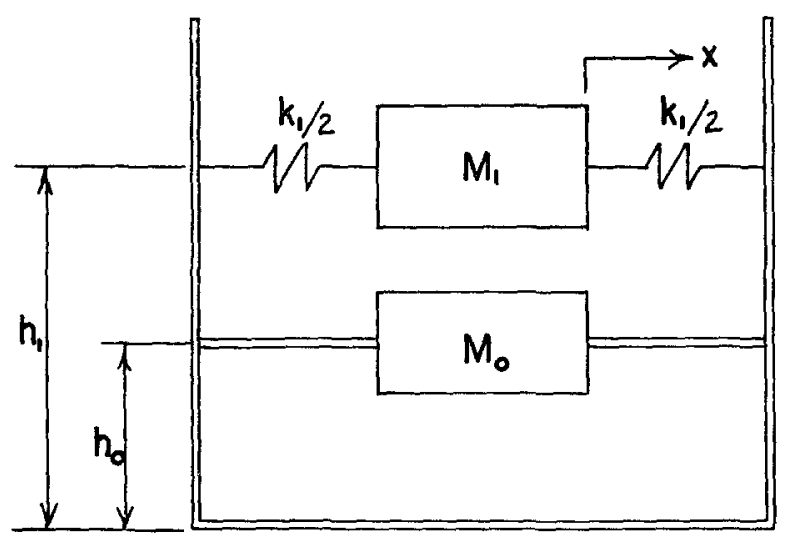

Fig. 1b

Frg. 1. Equivalent dynamic system for a water tank.

The tank with oscillating water surface is shown in 1a. The equivalent system is shown in $1 \mathrm{~b}$ where $M_{0}$ and $M_{1}$ produce dynamic forces equivalent to those produced by the water.

on the tank by $M_{0}$ and $M_{1}$ will be the same as would be exerted by the water in the tank of figure 1a.

The equivalent system is specified by the following quantities.., ${ }^{2}$ For cylindrical tank of radius $R$ and water depth $h$

$$
M_{0}=M \frac{\tanh 1.7 R / h}{1.7 R / h}
$$

1 Jacobsen, L. S., "Impulsive Hydrodynamics of Fluid Inside a Cylindrical Tank," Bull. Seism. Soc. Am., Vol. 39, 1949.

2 Housner, G. W., "Dynamic Pressures on Accelerated Fluid Containers," Bull. Seism. Soc. Am., Vol. 47, 1957 ." 


$$
\begin{aligned}
M_{1} & =M(0.6) \frac{\tanh 1.8 h / R}{1.8 h / R} \\
k_{1} & =5.4 \frac{M_{1}^{2}}{M} \frac{g h}{R^{2}} \\
h_{0} & =\frac{3}{8} h\left\{1+\alpha\left[\frac{M}{M_{1}}\left(\frac{R}{h}\right)^{2}-1\right]\right\} \\
\alpha & =1.33 \\
h_{1} & =h\left[1-0.185\left(\frac{M}{M_{1}}\right)\left(\frac{R}{h}\right)^{2}-0.56 \beta \frac{R}{h} \sqrt{\left(\frac{M R}{3 M_{1} h}\right)^{2}-1}\right] \\
\beta & =2.0 \\
T_{w} & =2 \pi \sqrt{\frac{M_{1}}{k_{1}}}=\text { a period of vibration } \\
M & =\text { total mass of water in the tank }
\end{aligned}
$$

For rectangular tanks of length $2 L$ and water depth $h$

$$
\begin{aligned}
M_{0} & =M \frac{\tanh 1.7 L / h}{1.7 L / h} \\
M_{1} & =M \frac{(0.83) \tanh 1.6 h / L}{1.6 h / L} \\
k_{1} & =3 \frac{M_{1}^{2}}{M} \frac{g h}{L^{2}} \\
h_{0} & =\frac{3}{8} h\left\{1+\alpha\left[\frac{M}{M_{1}}\left(\frac{L}{h}\right)^{2}-1\right]\right\} \\
\alpha & =1.33 \\
h_{1} & =h\left[1-\frac{1}{3} \frac{M}{M_{1}}\left(\frac{L}{h}\right)^{2}-0.63 \beta \frac{L}{h} \sqrt{0.28\left(\frac{M}{M_{1}} \frac{L}{h}\right)^{2}-1}\right] \\
\beta & =2.0
\end{aligned}
$$

If the heights $h_{0}$ and $h_{1}$ are to be determined on the basis of the dynamic fluid forces exerted on the walls of the tank only (not on the floor), the following values should be used for both cylindrical and rectangular tanks: $\alpha=0, \beta=1$.

A graph of the hyperbolic tangent (tanh $1.6 Z)$ is shown in figure 2.

The maximum oscillation of the fluid is related to the oscillation of the mass $M_{1}$ as follows. If $M_{1}$ is oscillating with displacement $x=A_{1} \sin \omega t$ the crest of the 
wave at the wall of the tank will oscillate up and down with displacement $y=d$ $\sin \omega t$ and for the cylindrical tank

$$
d=\frac{0.63 A_{1}\left(\frac{k_{1} R}{M_{1} g}\right)}{1-0.85 \frac{A_{1}}{R}\left(\frac{k_{1} R}{M_{1} g}\right)^{2}}
$$

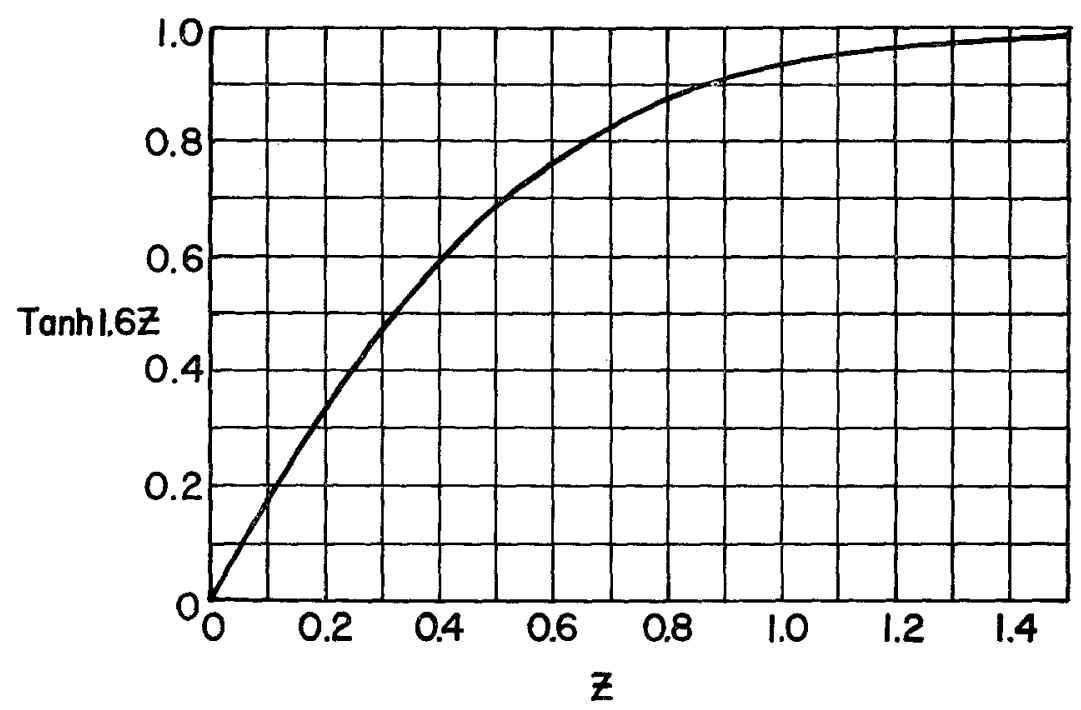

Fig. 2. Graph of $\tanh 1.6 Z$.

and for the rectangular tank

$$
d=\frac{0.84 A_{1}\left(\frac{k_{1} g}{M_{1} g}\right)}{1-\frac{A_{1}}{L}\left(\frac{k_{1} L}{M_{1} g}\right)^{2}}
$$

The foregoing equations give good results for amplitudes of vibration $d<0.2 L$ and $d<0.2 h$, although at the larger amplitudes a certain amount of nonlinearity is observed in the oscillations. ${ }^{3}$

As an example of the oscillations that may be induced by earthquake ground motion, consider a rectangular tank $50 \mathrm{ft}$ long and $10 \mathrm{ft}$ deep $(L=25 \mathrm{ft}, h=10$ $\mathrm{ft}$ ) that is resting on the ground. For this tank there is obtained a period of vibration of 6 seconds. For relatively strong ground motion with an undamped velocity spectrum value $S_{v}=2.0 \mathrm{ft} / \mathrm{sec}$, the displacement of the mass $M_{1}$ would have an amplitude

$$
A=2.0 \frac{T}{2 \pi}=1.9 \mathrm{ft}
$$

3 Conrad, A., "Hydrodynamic Forees Induced in Fluid Containers," Thesis, California Institute of Technology, 1956. It is also shown in this thesis that a cylindrical tank with a hemispherical bottom may be treated as a flat bottom tank of the same volume of water so far as computing the period and the dynamic forces. 
and the amplitude of the sloshing is found to be

$$
d=1.5 \mathrm{ft}
$$

It should be noted that there will also be a corresponding oscillation of the fluid at 90 degrees to the foregoing motion since the earthquake ground motion will have a component in that direction also.

\section{Elevated Water Tank}

An elevated water tank can be represented by the equivalent system shown in figure 3 . The spring constant $k$ represents the effective stiffness of the structure

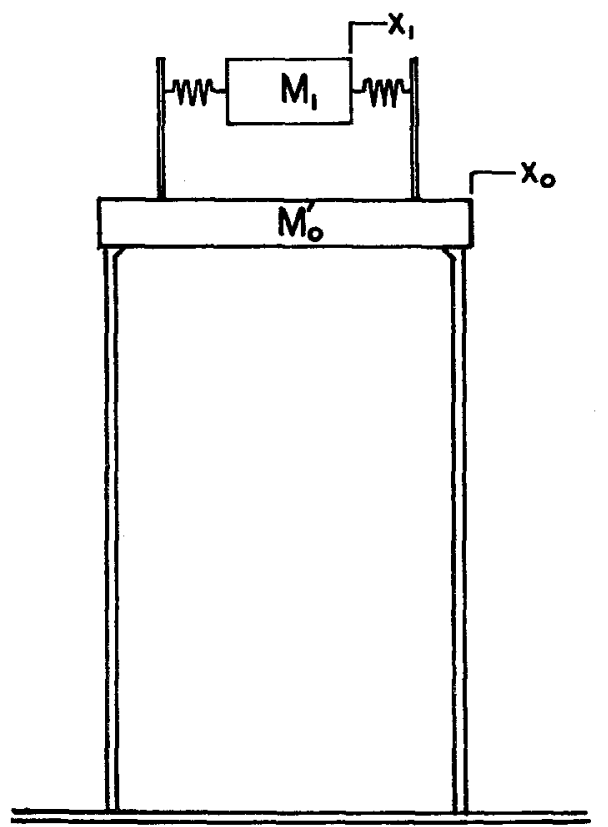

Fig. 3. Equivalent dynamic system for an elevated water tank.

and $M_{0}{ }^{\prime}$ is the equivalent mass of the structure plus $M_{0}$. As an illustration, a tank having $R=20 \mathrm{ft}$ and $h=15 \mathrm{ft}$ has a natural period of oscillation of water given by

$$
T=2 \pi \sqrt{\frac{M_{1}}{k_{1}}}=4.0 \mathrm{sec}
$$

where

$$
\begin{aligned}
& M_{1}=0.44 M=800,000 \mathrm{lb} / \mathrm{g} \\
& k_{1}=62,000 \mathrm{lb} / \mathrm{ft} \\
& M_{0}=0.35 M=640,000 \mathrm{lb} / \mathrm{g}
\end{aligned}
$$


If the mass and stiffness of the structure are such that

$$
\begin{aligned}
& k=700,000 \mathrm{lb} / \mathrm{ft} \\
& M_{0}^{\prime}=(640,000+160,000) \mathrm{lb} / \mathrm{g}=800,000 \mathrm{lb} / \mathrm{g}
\end{aligned}
$$

the two modes of vibration will have the shapes shown in fig. 4 , and the periods are respectively $T_{1}=4.2 \mathrm{sec}$ and $T_{2}=1.1$ second. It is seen that the period and forces of the first mode are closely approximated by the period and force of the fluid with an infinitely rigid supporting structure. The period and forces of the second mode

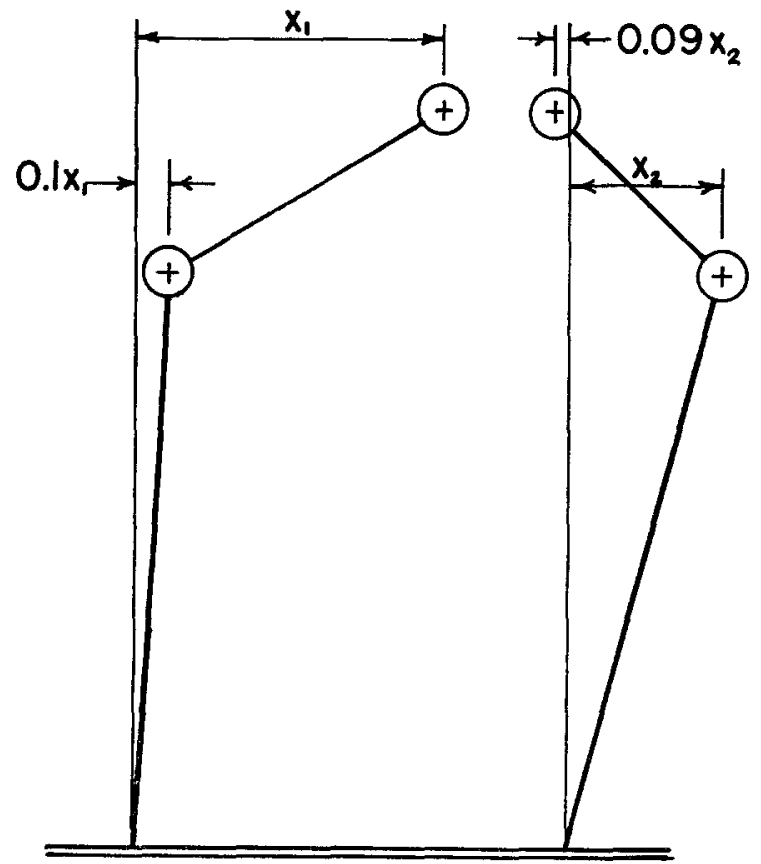

FIg. 4. The modes of vibration for an elevated water tank.

are essentially those of the mass $M_{0}^{\prime}$ alone disregarding the mass $M_{1}$. The maximum shear force produced by $M_{1}$ during ground motion with $S_{v}=2.0$ is

$$
F_{1}=\frac{2 \pi}{T} M_{1}=39,000 \mathrm{lb}
$$

with a period of 4.0 seconds. The maximum force produced by $M_{0}{ }^{\prime}$ is

$$
F_{2}=\frac{2 \pi}{T} M_{0}^{\prime}=140,000 \mathrm{lb}
$$

at a period of 1.1 seconds. Comparing with the total mass of the tank these forces are, respectively, equivalent to $2 \%$ and $7 \% \mathrm{~g}$. If the tank were completely full so 
that there could be no sloshing the period would be 1.7 sec and the force would be 220,000 pounds.

A comparison of the measured and the calculated periods of vibration of an elevated water tank are presented in a companion paper by W. K. Cloud.

As another example, consider a golf-ball-on-a-tee type of tank with radius $R$ $=20 \mathrm{ft}$ and half full of water. This may be treated as a cylindrical tank of the same radius and equal volume of water ${ }^{4}(R=20 \mathrm{ft}, h=13.3 \mathrm{ft})$, and the following values are obtained

$$
\begin{aligned}
& M=1,000,000 \mathrm{lb} / \mathrm{g} \\
& M_{0}=0.5 M=500,000 \mathrm{lb} / \mathrm{g} \\
& M_{1}=0.42 M=420,000 \mathrm{lb} / \mathrm{g} \\
& T=4.0 \mathrm{sec}
\end{aligned}
$$

If this is compared to the case of a completely full tank with $M_{0}=M$ and $M_{1}$ $=0$, it is seen that the maximum force to which the half-full tank is subjected may be significantly less than half the force to which the full tank is subjected if the maximum forces exerted by $M_{0}$ and $M_{1}$ happen not to coincide. The actual forces may be as little as $1 / 3$ of the forces anticipated on the basis of a completely full tank.

${ }^{4}$ Conrad, A., loc. cit.

California Institute of Temehology

Pasadena, California

Manuscript received April 12, 1962. 